\title{
Influência da paisagem nas assembleias de Scarabaeinae (Coleoptera: Scarabaeidae) em um ambiente agrícola no sul de Santa Catarina
}

\author{
Mariana Mrotskoski Niero * \\ Malva Isabel Medina Hernández \\ Universidade Federal de Santa Catarina, Campus Universitário Reitor João David Ferreira Lima \\ Trindade, CEP 88040-960, Florianópolis - SC, Brasil \\ * Autor para correspondência \\ mari.m.niero@gmail.com
}

Submetido em 29/11/2016

Aceito para publicação em 03/07/2017

\section{Resumo}

Reservas Legais visam à conservação de florestas nativas e processos ecológicos, podendo formar mosaicos heterogêneos na paisagem. Este trabalho objetivou descrever e comparar assembleias de besouros escarabeíneos coprófagos para melhor entender a distribuição das espécies dentro da paisagem. A amostragem foi realizada em Içara (SC), em 2014, em ambientes com matas nativas, plantações de eucalipto, de milho e em pastagens. Foram utilizadas 10 armadilhas de atração para insetos vivos em cada local, recolhidas após $48 \mathrm{~h}$. Foi coletado um total de 704 indivíduos e 16 espécies de Scarabaeinae, sendo as mais abundantes: Onthophagus catharinensis $(\mathrm{n}=213)$, Canthon smaragdulus $(\mathrm{n}=128)$ e Canthidium aff. trinodosum $(\mathrm{n}=112)$. Matas nativas obtiveram maiores riqueza e abundância. A similaridade de Bray-Curtis agrupou as assembleias dos ambientes de mata entre si ( $78 \%$ de similaridade), que diferiram das áreas com plantações de eucaliptos, assim como das assembleias de áreas abertas. Foram encontradas espécies com alto Valor Indicador Individual para ambientes de mata e de áreas abertas. A área de ligação entre matas teve grande importância, conectando áreas de mata nativa e auxiliando na conservação de espécies. Conforme o esperado, os resultados confirmam que ambientes mais preservados conservam maior biodiversidade, mas outros elementos da paisagem contribuem com a diversidade regional.

Palavras-chave: Bioindicadores; Conservação; Ecologia de paisagem; Fragmentação; Mata Atlântica

\section{Abstract}

Landscape's influence on assemblages of the Scarabaeinae (Coleoptera: Scarabaeidae) in an agricultural environment in the south of Santa Catarina. Legal reserves aim to conserve native forests and ecological processes, and are able to form heterogeneous mosaics in the landscape. This study aimed to describe and compare assemblages of coprophagous dung beetles to better understand the distribution of species within the landscape. Sampling was conducted in the municipality of Içara (SC) in 2014, within environments of native forests, plantations of eucalyptus and corn, and pastures. We used 10 traps to capture live insects at each site, collected after 48 hours. A total of 704 individuals were collected, including 16 Scarabaeinae species, where the most abundant were: Onthophagus catharinensis $(\mathrm{n}=213)$, Canthon smaragdulus $(\mathrm{n}=128)$ and Canthidium aff. trinodosum $(\mathrm{n}=112)$. Bray-Curtis similarity grouped the assemblages of forest environments with each 
other (78\% similarity) but not with those from areas with eucalyptus plantations or from open areas. Species with a high individual indicator value were found for forest environments and open areas. The connection area between forests was very important in connecting native forest areas and aiding in the conservation of species. As expected, the results confirm that more preserved environments conserve a greater biodiversity; however, other landscape components contribute to regional diversity.

Key words: Atlantic Forest; Bioindicators; Conservation; Fragmentation; Landscape ecology

\section{Introdução}

Paisagem, segundo Metzger (2001), é “um mosaico heterogêneo formado por unidades interativas, sendo esta heterogeneidade existente para pelo menos um fator, segundo um observador e numa determinada escala de observação". Partindo desse princípio, a ecologia de paisagens pode ser entendida como o estudo da estrutura, função e dinâmica de áreas heterogêneas formadas por ecossistemas interativos (FORMAN; GODRON, 1986), buscando entender a estrutura da comunidade pela dinâmica dos elementos da paisagem (METZGER, 2001), pois, dependendo das características das espécies estudadas, cada elemento de paisagem poderá ser percebido de forma diferenciada (METZGER, 2001; RICKLEFS, 2011). Desta forma, a ecologia de paisagens pode auxiliar na resolução de problemas ambientais, como no planejamento da expansão das fronteiras agrícolas, uso da terra, conservação da biodiversidade e sustentabilidade (METZGER, 2001; METZGER et al., 2007).

A destruição de ambientes naturais pela atividade humana para criação de áreas de pastagens ou monoculturas provoca alterações nos fatores abióticos, podendo ocasionar, com isso, uma mudança na composição e estrutura das comunidades ou acarretar perda de espécies não adaptadas às novas condições ambientais (BARLOW et al., 2007). Atualmente, entre as ameaças mais severas à biodiversidade encontram-se: a destruição de habitats, a fragmentação da paisagem, a intensificação dos usos do solo e as invasões biológicas (SALA et al., 2000). A expansão agrícola pode causar a perda de espécies por destruição e mudança de habitat, redução das populações, perda de migração (com efeitos demográficos e genéticos), alteração do microclima e imigração de espécies exóticas (THOMAZINI; THOMAZINI, 2000). Além disso, a percolação (capacidade das espécies de atravessar a paisagem) e os efeitos de borda em fragmentos são fatores de possíveis extinções locais (METZGER et al., 2007; RIBEIRO et al., 2009).

O tamanho dos fragmentos, suas formas e a conectividade entre eles são fatores muito importantes na manutenção da diversidade (RIBEIRO et al., 2009; METZGER, 2010), e os padrões dos diversos elementos da paisagem, como manchas, corredores e matrizes, são muito utilizados em estudos de conservação. As Reservas Legais, previstas no Código Florestal de 2012 (Lei no 12.651) (BRASIL, 2012), são fragmentos florestais que funcionam como importantes elementos da paisagem, já que formam mosaicos heterogêneos com diferentes habitats para os organismos, mesmo em pequenas escalas, propiciando importantes serviços ambientais, como ciclagem de nutrientes, controle de pragas, aumento da polinização e da produtividade de algumas culturas (DE MARCO; COELHO, 2004). Alguns organismos podem auxiliar questões de conservação e manejo, por serem considerados bioindicadores, como os insetos, que possuem relativa plasticidade no uso de diversos ambientes, respondem rapidamente às alterações ambientais, são especialistas em recursos e estão presentes em áreas pequenas e fragmentadas (BROWN, 1997; BARLOW et al., 2007; GARDNER et al., 2008b). Entre os insetos, os besouros escarabeíneos são frequentemente utilizados como indicadores ecológicos, pois as mudanças ambientais alteram também a estrutura e a composição das espécies dentro das comunidades, com diminuição de riqueza, abundância e diversidade (KLEIN, 1989; HALFFTER; FAVILA, 1993; DAVIS et al., 2001; GARDNER et al., 2008a; CAMPOS; HERNÁNDEZ, 2014; HERNÁNDEZ et al., 2014). Participam, enquanto larvas e adultos, na ciclagem de nutrientes e no ciclo de decomposição da matéria orgânica ao se alimentarem de fezes (coprofagia) e animais em decomposição (necrofagia), cumprindo uma relevante função ecossistêmica (HALFFTER; MATTHEWS, 1966; HALFFTER; EDMONDS, 1982), 
contribuindo significativamente em processos de aeração e fertilidade do solo (SLADE et al., 2007; BATILANIFILHO, 2015), possuindo alta sensibilidade às mudanças ambientais por conta da íntima relação com o meio e os recursos disponíveis (HALFFTER; FAVILA, 1993; BARLOW et al., 2007; GARDNER et al., 2008b; HERNÁNDEZ; VAZ-DE-MELLO, 2009) e respondendo negativamente à destruição, fragmentação e isolamento de florestas tropicais (KLEIN, 1989; HALFFTER et al., 1992). Muitas espécies de Scarabaeinae são altamente específicas quando se trata do habitat e não estendem suas populações para áreas abertas (KLEIN, 1989; ALMEIDA; LOUZADA, 2009), podendo diminuir ou desaparecer localmente (HERNÁNDEZ; VAZ-DEMELLO, 2009; HERNÁNDEZ et al., 2014), diminuindo, assim, o pool de espécies para outras áreas da paisagem (KORASAKI et al., 2013).

Partindo da hipótese de que áreas mais conservadas conseguem abrigar maior riqueza de espécies e abundância de organismos, o presente estudo teve por objetivos descrever e analisar a diversidade de besouros escarabeíneos em uma paisagem predominantemente agrícola no sul do Brasil, além de verificar como as espécies se distribuem espacialmente em diferentes elementos da paisagem incluindo manchas de mata (representadas por Reservas Legais) e agroecossistemas (eucalipto, pastagem e plantio de milho). Ainda, buscouse avaliar quais espécies de Scarabaeinae são associadas a algum dos tipos de ambientes amostrados.

\section{Material e Métodos}

\section{Área de estudo}

O estudo foi realizado no município de Içara $\left(28^{\circ} 68^{\prime} \mathrm{S}\right.$ e $4^{\circ} 28^{\prime} \mathrm{O}$, altitude média de $\left.31 \mathrm{~m}\right)$, próximo à divisa com o município de Criciúma, na região do extremo sul de Santa Catarina, em fragmentos de paisagem localizados em propriedades particulares. Içara faz parte da Associação dos Municípios da Região Carbonífera (AMREC). O clima da região, segundo a classificação climática de Köppen, é do tipo Cfa (mesotérmico, úmido e com verão quente) com temperaturas médias variando entre 9 e $30^{\circ} \mathrm{C}$, e a pluviosidade do litoral sul de Santa
Catarina variando de 1220 a $1660 \mathrm{~mm}$ de precipitação total anual, com umidade relativa em torno de $80 \%$ (BACK, 2009). O município pertence ao bioma Mata Atlântica (IBGE, 2014), com Floresta Ombrófila Densa (quase extinta na região atualmente).

A região das coletas é um grande mosaico agropecuário e de fragmentos de mata, sendo estes nativos (primários ou secundários) ou exóticos (plantações de eucalipto); isso porque, com a obrigatoriedade de se conservar Reservas Legais, muitos proprietários reservam os fundos do terreno, que sendo, em geral, de pequeno porte, unem-se, formando manchas contínuas de mata. Todos esses fragmentos, em uma escala mais ampla, formam uma heterogeneidade de habitats possíveis para as espécies (Figura 1). Os elementos da paisagem amostrados foram: manchas de mata maior e menor, área de ligação entre mata maior e menor, área de plantação de eucalipto contínua à mata e mancha de eucalipto isolada, área de pastagem e plantação agrícola de milho (Figura 1).

\section{Coleta de dados}

A coleta dos insetos foi realizada durante o mês de dezembro de 2014 por meio de armadilhas de queda com iscas de atração (LOBO et al., 1988). Foram montadas 10 armadilhas em cada área de coleta (unidade de paisagem pré-determinada, mostrada na Figura 1), distantes aproximadamente $10 \mathrm{~m}$ entre si, iscadas com $20 \mathrm{~g}$ de fezes humanas cada. As armadilhas, 70 no total, consistiram em potes plásticos de $15 \mathrm{~cm}$ de diâmetro e 7,5 cm de altura, enterradas ao nível do solo. A tampa de plástico do pote possuía uma abertura para que os besouros conseguissem entrar, e o fundo do pote foi preenchido com terra, serapilheira e a isca.

As armadilhas foram revistadas após $48 \mathrm{~h}$, com identificação dos indivíduos realizada in situ com auxílio de uma coleção de referência (espécies identificadas que tinham sido amostradas em coleta piloto na mesma área $\mathrm{e}$ em outras regiões litorâneas do estado). Grande parte dos indivíduos amostrados foi devolvida ao ambiente após as coletas de dados. Alguns indivíduos de cada espécie, e os que não puderam ser identificados, foram mortos por congelamento. Logo após, esses animais foram depositados em álcool $70^{\circ} \mathrm{GL}$ e, posteriormente, levados 
FIGURA 1: Mapa de Santa Catarina e município de Içara, com destaque para os locais onde foram realizadas as coletas de Scarabaeinae em dezembro de 2014. A: mata menor, B: mata maior, C: área de ligação entre matas, D: mancha de eucalipto isolada da mata, E: mancha de eucalipto contínua à mata, F: pastagem, G: cultivo de milho.

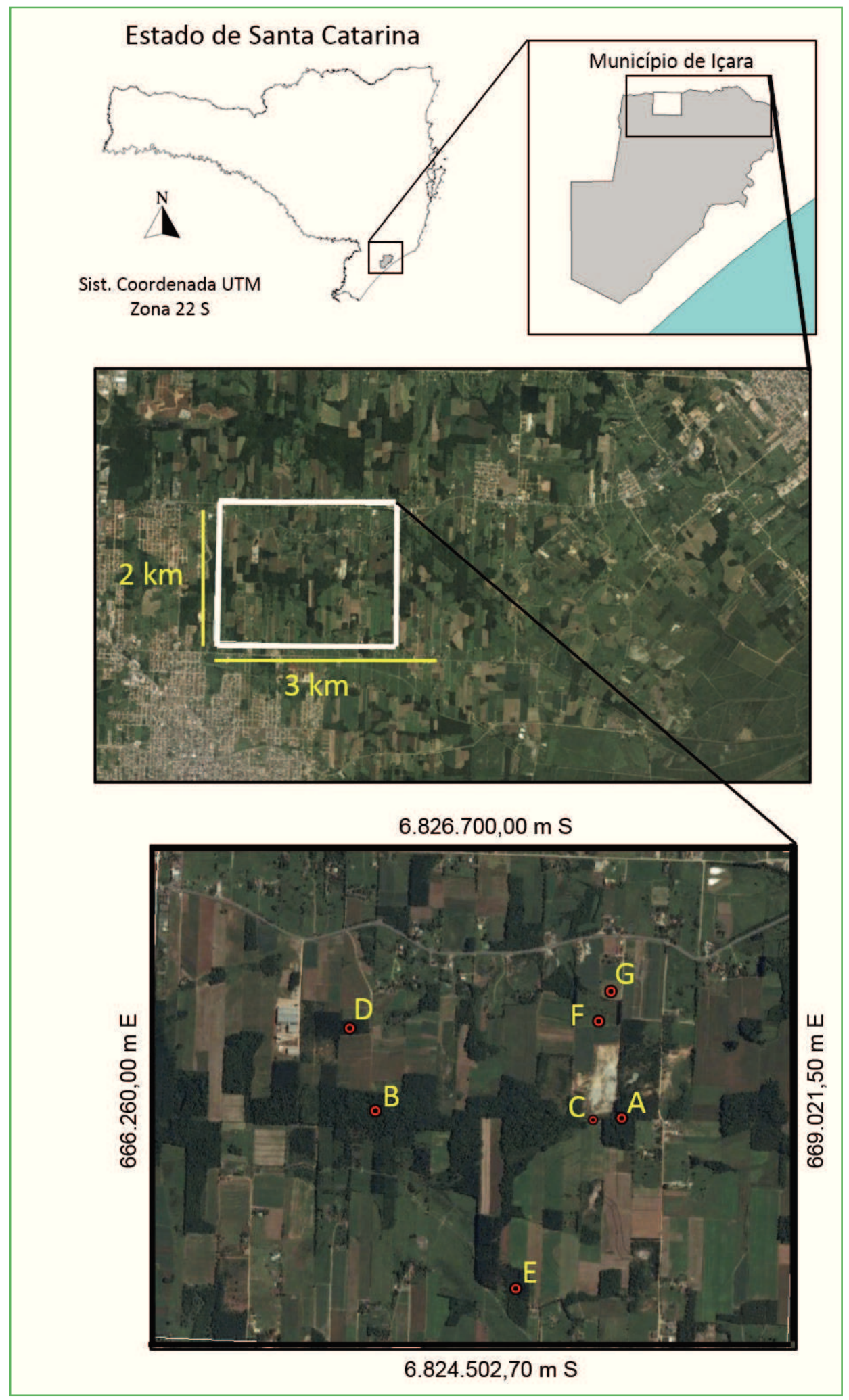


para a confirmação da identificação no Laboratório de Ecologia Terrestre Animal (LECOTA), na Universidade Federal de Santa Catarina (UFSC), em Florianópolis. Os insetos levados ao laboratório foram triados, alfinetados, secos em estufa (por três dias a temperatura de $45^{\circ} \mathrm{C}$ ), identificados e depositados na Coleção Entomológica do Centro de Ciências Biológicas da UFSC.

\section{Análise dos dados}

Para a descrição das assembleias foi analisada a distribuição e a abundância dos indivíduos de cada espécie. $\mathrm{O}$ esforço amostral foi mostrado por uma curva de acumulação de espécies para cada área, e o estimador de riqueza Jackknife 1 foi utilizado, ambas as análises feitas com o programa EstimateS v.9.1.0 (COLWELL, 2013). Os índices de diversidade de Shannon (H') e de equitabilidade (Pielou) foram calculados para descrever a diversidade $\alpha$ de cada área amostral no programa Primer $6 \beta$ (CLARKE; GORLEY, 2006). O mesmo programa foi utilizado para calcular o índice quantitativo de similaridade (Bray-Curtis, cujos grupos foram testados por simproftest) e comparar as assembleias dos elementos da paisagem (diversidade $\beta$ ).

No programa PC-ORD 5.10(McCUNE; MEFFORD, 2006) foi calculado o Valor Indicador Individual (IndVal) para apontar possíveis espécies associadas aos diferentes habitats amostrados. Esta análise combina a especificidade da espécie ao elemento da paisagem na qual ocorre (através de padrões de abundância relativa) e sua fidelidade em relação a esse elemento (DUFRÊNE; LEGENDRE, 1997). Para a realização da análise, foram formados grupos com fitofisionomias similares e agrupadas as áreas em uma escala maior: Mata (englobando Mata Maior e Menor), Eucalipto (tanto Contínuo como Isolado) e Área Agrícola (com os locais mais abertos, compreendendo Área de Ligação, Pastagem e Plantação).

\section{Resultados}

Foram coletados 704 indivíduos e 16 espécies (Tabela 1; Figura 2), dos quais 187 foram mortos para

TABELA 1: Lista de espécies de Scarabaeinae, riqueza, abundância, diversidade e equitabilidade das assembleias coletadas em diferentes elementos da paisagem em dezembro de 2014 em Içara, Santa Catarina. MMa: Mata Maior; MMe: Mata Menor; AL: Área de Ligação; ECo: Eucalipto Contínuo à mata maior; EIs: Eucalipto Isolado; Pas: Pastagem; Pla: Plantação de milho.

\begin{tabular}{l|ccccccc|c}
\multicolumn{1}{c|}{ Espécie } & MMa & MMe & AL & ECo & EIs & Pas & Pla & Total \\
\hline Canthidium aff. dispar Harold, 1867 & - & 1 & - & 1 & - & - & - & 2 \\
Canthidium aff. trinodosum (Boheman, 1858) & 42 & 68 & 2 & - & - & - & - & 112 \\
Canthon lividus seminitens Harold, 1867 & - & - & 22 & - & - & 1 & 8 & 31 \\
Canthon rutilans cyanescens Harold, 1868 & - & - & 1 & 2 & 2 & - & - & 5 \\
Canthon smaragdulus (Fabricius, 1781) & 76 & 38 & 3 & 1 & 10 & - & - & 128 \\
Coprophanaeus saphirinus (Sturm, 1826) & - & 1 & - & - & - & - & - & 1 \\
Deltochilum morbillosum Burmeister, 1848 & - & 1 & - & - & - & - & - & 1 \\
Deltochilum multicolor Balthasar, 1939 & - & - & 3 & - & - & - & - & 3 \\
Dichotomius assifer (Eschscholtz, 1822) & 2 & - & - & - & - & - & - & 2 \\
Dichotomius mormon (Ljungh, 1799) & 58 & 23 & 1 & - & - & - & - & 82 \\
Dichotomius nisus (Olivier, 1789) & - & - & - & - & - & 9 & - & 9 \\
Dichotomius sericeus (Harold, 1867) & 13 & 23 & - & 7 & - & - & - & 43 \\
Ontherus sulcator (Fabricius, 1775) & - & - & - & 1 & - & 2 & - & 3 \\
Onthophagus catharinensis Paulian, 1936 & 165 & 41 & 6 & - & 1 & - & - & 213 \\
Phanaeus splendidulus (Fabricius, 1781) & 45 & 7 & - & - & - & - & - & 52 \\
Trichillum externepunctatum Borre, 1880 & - & - & 9 & - & - & 8 & - & 17 \\
\hline Riqueza & 7 & 9 & 8 & 5 & 3 & 4 & 1 & 16 \\
Abundância & 401 & 203 & 47 & 12 & 13 & 20 & 8 & 704 \\
Diversidade de Shannon-Wiener (log $\mathbf{e}$ ) & 1,580 & 1,691 & 1,584 & 1,234 & 0,687 & 1,106 & 0 & - \\
Equitabilidade de Pielou & 0,812 & 0,770 & 0,762 & 0,767 & 0,625 & 0,798 & 0 & - \\
\hline
\end{tabular}


FIGURA 2: Espécies de escarabeíneos coletadas em diferentes elementos da paisagem em dezembro de 2014 em Içara, Santa Catarina. A: Canthidium aff. dispar, B: Canthidium aff. trinodosum, C: Canthon lividus seminitens, D: Canthon rutilans cyanescens, E: Canthon smaragdulus, F: Coprophanaeus saphirinus, G: Deltochilum morbillosum, H: Deltochilum multicolor, I: Dichotomius assifer, J: Dichotomius mormon, K: Dichotomius nisus, L: Dichotomius sericeus, M: Ontherus sulcator, N: Onthophagus catharinensis, O: Phanaeus splendidulus, P: Trichillum externepunctatum.

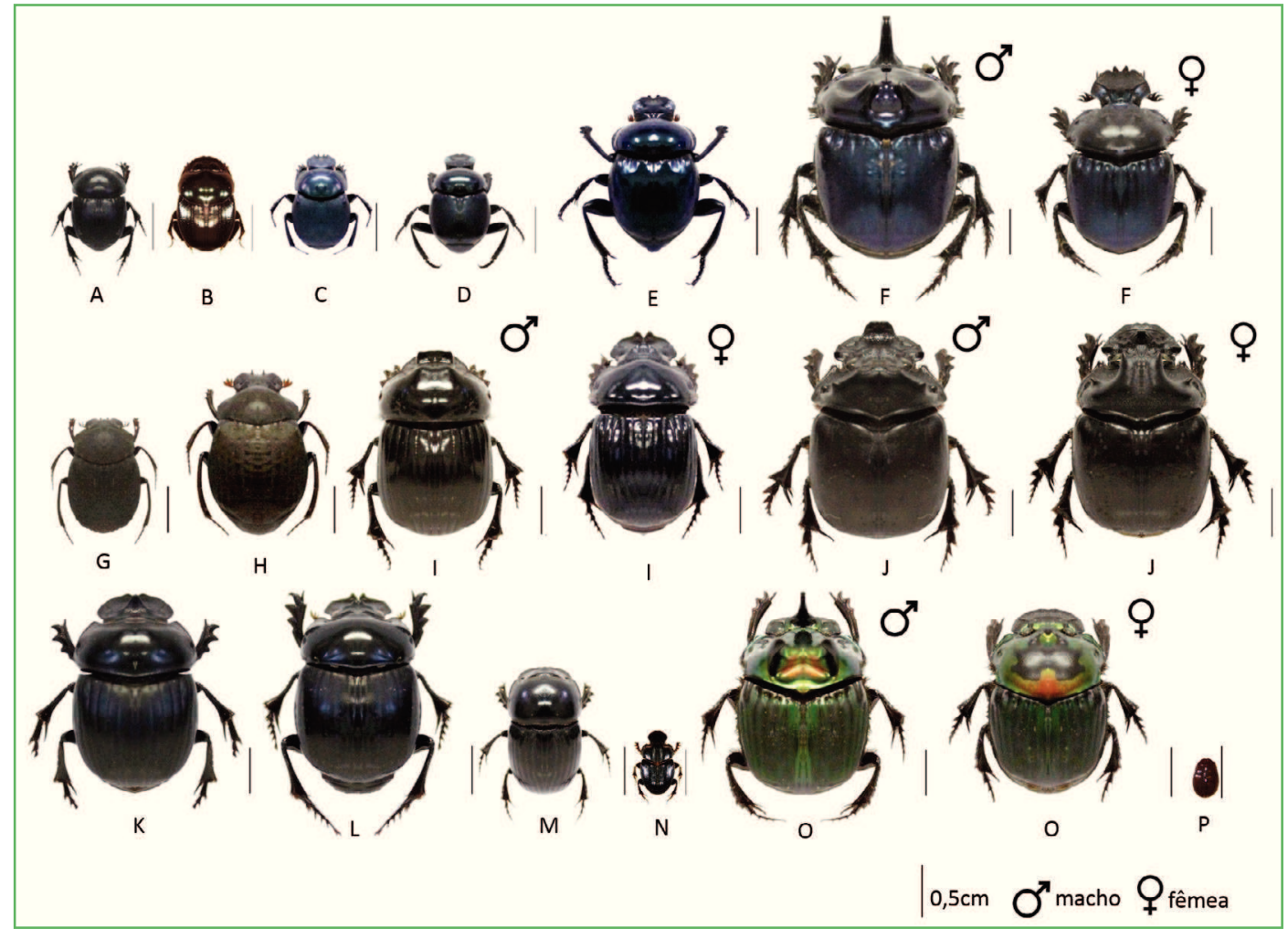

Fonte: Acervo do Laboratório de Ecologia Terrestre Animal (fotos de Mariana Mrotskoski Niero).

confirmar a identificação e os outros 517 foram soltos após contagem e identificação.

As espécies que tiveram as maiores abundâncias foram Onthophagus catharinensis (30\% dos espécimes coletados), Canthon smaragdulus (18\%), Canthidium aff. trinodosum (16\%) e Dichotomius mormon (12\%). Canthidium aff. dispar, Canthon rutilans cyanescens, Coprophanaeus saphirinus, Deltochilum morbillosum, Deltochilum multicolor, Dichotomius assifer, Dichotomius nisus e Ontherus sulcator tiveram baixa abundância, aparecendo em, no máximo, três áreas (Tabela 1).

As matas nativas tiveram as maiores riquezas, com Mata Menor apresentando nove espécies, seguida pela Área de Ligação, com oito, e Mata Maior, com sete (Tabela 1). As áreas de mata se destacaram pela elevada abundância de escarabeíneos, tendo sido coletado na Mata Maior 57\% $(\mathrm{N}=401)$ de todos os indivíduos, quase o dobro da Mata Menor $(\mathrm{N}=203)$, que ficou em segundo lugar, com $29 \%$ do total. O índice de diversidade de Shannon-Wiener demonstrou que Mata Menor $\left(H^{\prime}=1,69\right)$, Mata Maior e Área de Ligação (ambos com $\left.H^{\prime}=1,58\right)$ foram as áreas com maiores valores de diversidade, e a área de Eucalipto Isolado obteve o menor valor $\left(H^{\prime}=0,687\right)$. O índice de equitabilidade de Pielou mostrou valores semelhantes entre as assembleias das diversas áreas, embora tenha apresentado um valor um pouco mais alto para Mata Maior e mais baixo para Eucalipto Isolado (Tabela 1).

As curvas de acumulação de espécies mostraram que as coletas nas diferentes áreas tenderam a estabilizar sua riqueza de espécies para três áreas: Mata Maior, Eucalipto Isolado e Pastagem (Figura 3). As áreas de Ligação (64\% das possíveis espécies do local foram coletadas) e de Eucalipto Contínuo (65\% da riqueza provável amostrada) tiveram menor suficiência amostral, 
já que a curva tende a subir mais abruptamente, diferentemente das demais áreas. Mata Maior teve $89 \%$ de suas espécies registradas, Mata Menor, 78\%, Eucalipto Isolado, 77\% e Pastagem, 82\%.

FIGURA 3: Curva de acumulação de espécies para coletas de Scarabaeinae realizadas em dezembro de 2014 em Içara, Santa Catarina. MMa: Mata Maior; MMe: Mata Menor; AL: Área de Ligação; ECo: Eucalipto Contínuo à mata maior; EIs: Eucalipto Isolado; Pas: Pastagem.

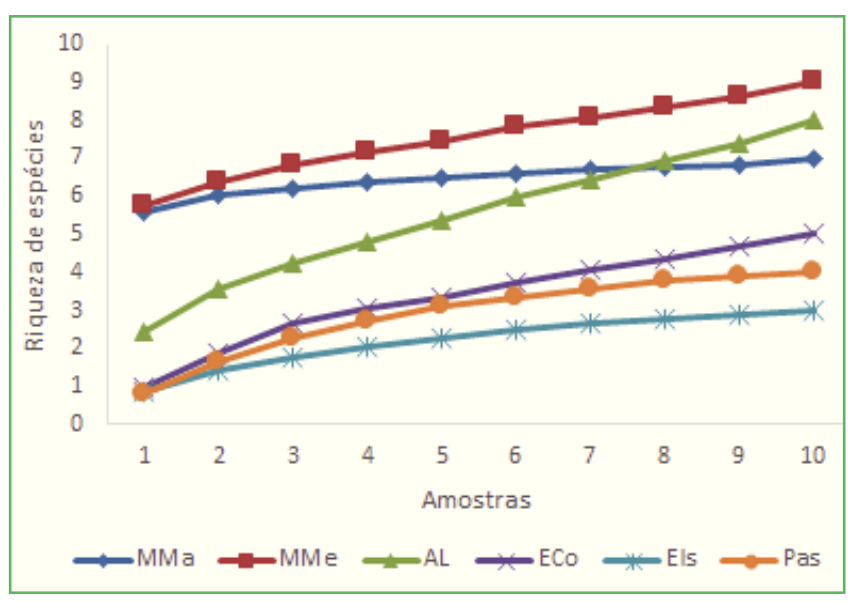

Com relação à similaridade das assembleias de escarabeíneos, houve formação de três grupos distintos: Área de Ligação e Plantações (com similaridade de 43\% entre si) formaram um grupo, juntamente com a área de Pastagem, similar em apenas $10 \%$ em relação aos demais locais (essas três áreas são abertas e a presença e/ou abundância de $C$. lividus seminitens teve influência no resultado deste grupo) (Figura 4). As áreas de eucalipto agruparam-se com um valor de $37 \%$ de similaridade e as duas matas (áreas florestais) tiveram seis espécies em comum, com assembleias similares em 78\%.

A partir dos grupos com fitofisionomias similares (Mata, Eucalipto e Área Agrícola), os valores da análise IndVal mostraram que as espécies $D$. mormon, $C$. aff. trinodosum, O. catharinensis, C. smaragdulus, $P$. splendidulus e $D$. sericeus são boas representantes de Mata, com altos valores indicadores, sendo espécies praticamente restritas a este ambiente; C. lividus seminitens e $T$. externepunctatum estão associadas a Áreas agrícolas, mais abertas; Eucalipto não obteve nenhuma espécie com alto IndVal, mostrando que não há espécies exclusivas deste ambiente (Tabela 2).

FIGURA 4: Dendrograma do Índice de Similaridade de Bray-Curtis para assembleias de Scarabaeinae coletadas em dezembro de 2014 em elementos da paisagem em Içara, Santa Catarina.

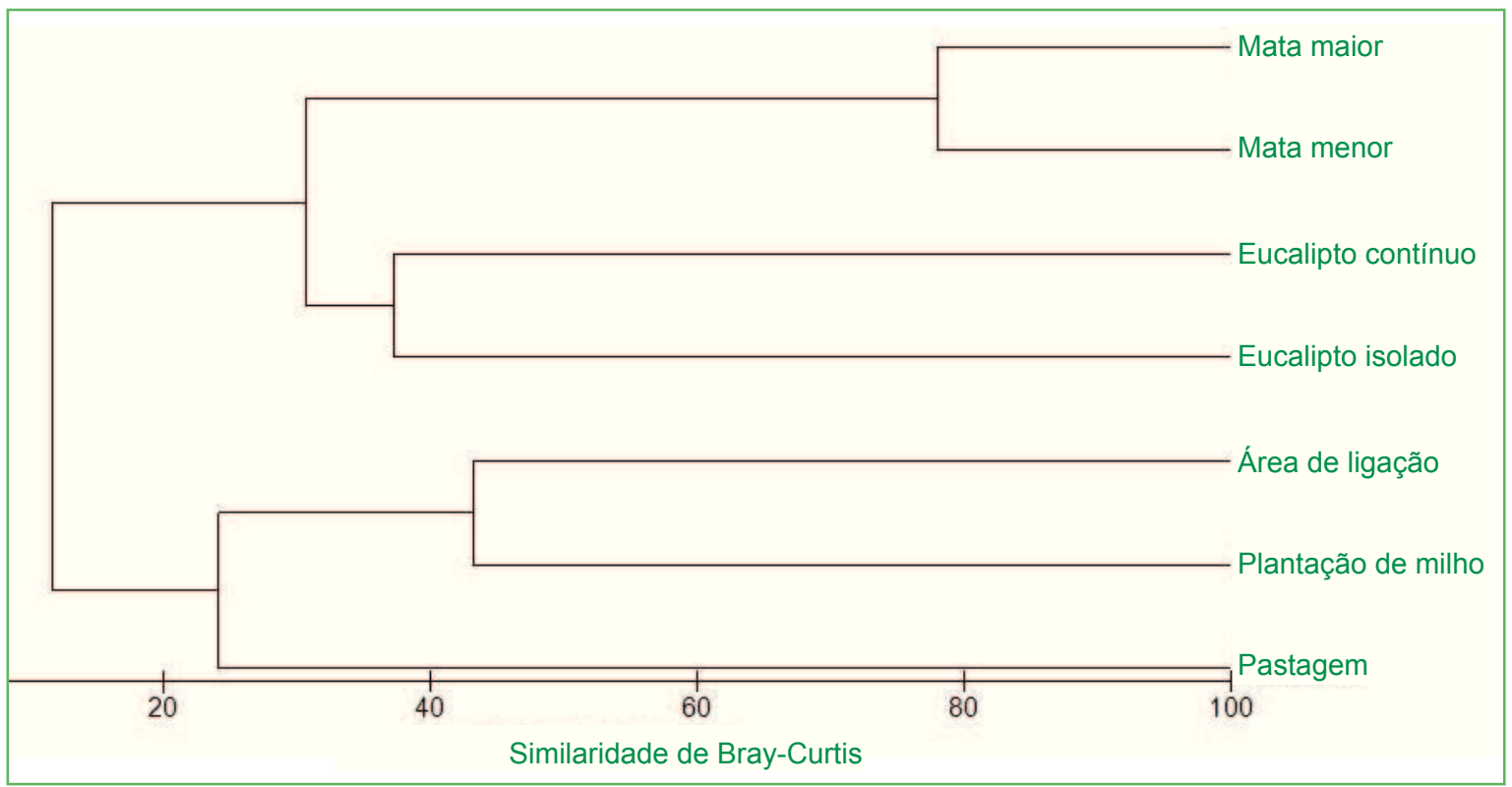


TABELA 2: Valores do teste de IndVal para as espécies de escarabeíneos coletadas em Içara, Santa Catarina, em dezembro de 2014 (IndVal > 30 e p < 0,05). M: Mata; E: Eucalipto; A: Área Agrícola.

\begin{tabular}{lccc}
\hline \multicolumn{1}{c}{ Espécie } & Local & $\begin{array}{c}\text { Valor } \\
\text { IndVal }\end{array}$ & p \\
\hline Dichotomius mormon & $\mathrm{M}$ & 99,2 & 0,0002 \\
Canthidium aff. trinodosum & $\mathrm{M}$ & 98,8 & 0,0002 \\
Onthophagus catharinensis & $\mathrm{M}$ & 97,6 & 0,0002 \\
Canthon smaragdulus & $\mathrm{M}$ & 89,8 & 0,0002 \\
Phanaeus splendidulus & $\mathrm{M}$ & 75,0 & 0,0002 \\
Dichotomius sericeus & $\mathrm{M}$ & 62,8 & 0,0002 \\
Canthon lividus seminitens & $\mathrm{A}$ & 46,7 & 0,0002 \\
Trichillum externepunctatum & $\mathrm{A}$ & 33,3 & 0,0002 \\
\hline
\end{tabular}

\section{Discussão}

O histórico de ocupação da região onde foi realizado o trabalho (Criciúma e municípios vizinhos), no extremo sul de Santa Catarina, envolve no início do século XX o ciclo do carvão, que gerou impactos profundos, como o aumento populacional e a demanda por alimento (SIZENANDO, 2011). Desta forma, aumentaram as áreas cultiváveis e os remanescentes de Mata Atlântica se reduziram, basicamente, às Reservas Legais nas cidades mais litorâneas, como em Içara, tornando o macro ambiente muito modificado e degradado.

O presente estudo, focado na análise espacial, teve as áreas de coleta próximas entre si, o que permitiria a passagem dos espécimes pelos diferentes ambientes, já que algumas espécies podem voar quase $1 \mathrm{~km}$ em períodos curtos de tempo (SILVA; HERNÁNDEZ, 2015). Mesmo assim, os besouros não foram encontrados de forma homogênea dentro da paisagem e houve preferências de algumas espécies por determinados habitats, e isso se traduziu na heterogeneidade espacial das assembleias em relação à composição, riqueza e abundância de escarabeíneos.

As maiores riquezas de besouros escarabeíneos observadas em áreas de mata em relação a áreas com plantações de eucalipto podem ser explicadas devido a menor complexidade vegetal (e, por consequência, animal); homogeneidade do habitat; animais visitantes; menor diversidade de compostos orgânicos na serapilheira; condições microclimáticas desfavoráveis para algumas espécies (altas temperaturas, radiação solar e menor cobertura do solo para as larvas) (GARDNER et al., 2008b; HERNÁNDEZ; VAZ-DE-MELLO, 2009; RIEFF et al., 2010; BRAGA et al., 2013; CAMPOS; HERNÁNDEZ, 2013; SILVA et al., 2013). Gardner et al. (2008b), trabalhando na Amazônia, defendem que, embora não possam ser considerados "desertos verdes", pois as espécies podem sobreviver em locais próximos à mata, áreas de eucalipto abrigam recursos limitados para as espécies desses locais.

Quando comparada às matas, área de pastagem também possui menor riqueza e abundância de escarabeíneos (MEDRI; LOPES, 2001; SILVA et al., 2014), sugerindo a dificuldade das espécies em colonizar essas áreas e, por isso, a menor diversidade em relação às matas. Mesmo assim, a diversidade em áreas de pecuária é normalmente maior que em agricultura (RESENDE; 2012). Porém, vale ressaltar que algumas espécies são altamente especializadas em áreas abertas (como no caso de Dichotomius nisus e Trichillum externepunctatum) e desempenham importantes papéis no sistema pecuário (FARIAS, 2016), participando da ciclagem de nutrientes (HALFFTER; MATTHEWS, 1966; HANSKI; CAMBEFORT, 1991), aumentando a velocidade de decomposição das fezes do gado, fazendo parte da dinâmica da paisagem na área estudada (NICHOLS et al., 2008). Além disso, as áreas agrícolas também sofrem perda na riqueza e na abundância de espécies (MENDEZ, 2007; RESENDE, 2012; KORASAKI et al., 2013), pois as fezes nesses ambientes de agricultura dessecam mais rapidamente (KLEIN, 1989) e, assim, apenas espécies adaptadas a essas condições conseguem obtê-las (HANSKI; CAMBEFORT, 1991).

A alta diversidade encontrada na área de Ligação pode estar no fato de ela ficar muito próxima das demais áreas de coleta e, por isso, ser um ambiente intermediário, onde várias espécies coexistem e se encontram. A referida área possui vegetação praticamente arbustiva e herbácea, não podendo sustentar, por si só, uma assembleia tão diversa e, assim, os espécimes provêm, provavelmente, das matas circundantes e dos habitats de pastagem, eucalipto e plantações. Esse argumento é sustentado ainda pela similaridade de Bray-Curtis, que agrupou a área de Ligação com Pastagem e Plantação 
de milho (todas áreas mais abertas). $\mathrm{O}$ fato de as áreas não serem isoladas ou distantes umas das outras pode favorecer o fluxo de indivíduos, mas a alta diversidade registrada nesse local eleva sua importância como fator de dinâmica da assembleia de escarabeíneos.

As espécies que puderam ser associadas a algum tipo de ambiente - possuindo alto Valor Indicador Individual (IndVal) - foram as de ambientes de mata ou de áreas mais abertas, sem espécies representantes de áreas de eucalipto, devido ao fato de que as espécies presentes nesse habitat costumam ser generalistas em relação à área ou são espécies "turistas" (AUDINO et al., 2011). Isso reflete o fato de haver espécies de áreas abertas ou pastagens diferentes das de áreas de mata, sendo independentes umas das outras (HALFFTER; ARELLANO, 2002). As espécies indicadoras de determinados ambientes se especializam e possuem um nicho mais estreito (KLEIN, 1989; ALMEIDA; LOUZADA, 2009), atentando para a importância de estratégias de conservação que visem à preservação de remanescentes de mata para a sobrevivência de espécies que possuem restrições de habitats. Trichillum externepunctatum já foi descrita como indicadora de áreas abertas (SCHIFFLER et al., 2003; SILVA et al., 2014; FILGUEIRAS et al., 2015) e C. lividus seminitens, também indicadora de áreas abertas, foi associada à área de eucalipto em Simões (2013). Dichotomius mormon foi associada a áreas de matas neste estudo, assim como no trabalho de Filgueiras et al. (2015), porém, Simões (2013) teve a espécie como indicadora de áreas de pinus. Canthidium aff. trinodosum, indicador de áreas de mata é uma espécie muito comum nesses ambientes (MEDRI; LOPES, 2001; HERNÁNDEZ; VAZ-DEMELLO, 2009; SIMÕES, 2013; SILVA et al., 2013). Ontherus sulcator pode ser encontrada em ecossistemas mais degradados (SILVA et al., 2013), assim como em plantações de espécies exóticas (AUDINO et al., 2011). Canthon rutilans cyanescens, D. multicolor, $D$. sericeus e $O$. sulcator foram algumas das espécies registradas por Bett et al. (2014) em áreas de regeneração de antigas minas de carvão, com diferentes idades de reflorestamento, em Lauro Müller, Santa Catarina. Claramente, essas espécies conseguem habitar áreas de menor complexidade ambiental e aclimatar-se às condições ambientais que não são favoráveis a outras espécies. A riqueza de espécies depende, portanto, também do tipo de distúrbio no ambiente (DAVIS et al., 2001).

A heterogeneidade horizontal da paisagem favorece a sobrevivência e coexistência das espécies que ocorrem em áreas florestais e abertas, contribuindo para a diversidade regional (HALFFTER; ARELLANO, 2002). Estudos utilizando bioindicadores, como os escarabeíneos, podem auxiliar no planejamento das áreas a serem protegidas, além das Reservas Legais, conservando as espécies e assegurando a permanência delas na paisagem. Agro-mosaicos compostos por poucas e grandes manchas de floresta, pequenos fragmentos florestais diferentemente afetados pela ação humana e sob efeitos de borda, florestas secundárias e monoculturas de espécies exóticas ou pastagens são a realidade mais comum dos remanescentes de Mata Atlântica nas paisagens atuais, havendo diferenças de biodiversidade entre esses ambientes, assim como diferentes grupos de espécies adaptadas a eles (TABARELLI et al., 2010). Os escarabeíneos, por serem indicadores ecológicos e terem tido alta diversidade nos locais amostrados, indicam uma alta diversidade também de outros animais, principalmente mamíferos, já que estes são os maiores provedores de recursos desses besouros. Técnicas de manejo e conservação protegem a comunidade biótica em níveis de espécie, habitat, populações e genes, além das interações ecológicas, processos e algumas práticas culturais historicamente associadas aos ecossistemas (SOULÉ, 1991). Assim, a conexão das Reservas Legais com outras áreas de preservação aumentaria a percolação na paisagem e diminuiria efeitos de borda, podendo ser um recurso utilizado em técnicas de manejo e conservação ambiental (RIBEIRO et al., 2009; TABARELLI et al., 2010). Segundo as estratégias propostas por Soulé (1991), as que poderiam ser implementadas em Içara e municípios vizinhos são in situ, inter situ e de restauração ecológica, a fim de melhor preservar os poucos e pequenos remanescentes de Mata Atlântica que, por lei, ainda estão mantidos na região, e que abrigam uma alta diversidade de organismos que precisam estritamente desses ambientes para sobreviver.

A Mata Atlântica abriga uma grande diversidade de organismos, sendo considerada um hotspot, estando entre 
os biomas mais ameaçados do mundo, principalmente devido ao desmatamento, que pode ser causado pelo aumento da urbanização e industrialização, de áreas de pastagem, do plantio de eucalipto e do desenvolvimento de monoculturas (TABARELLI et al., 2010). Nosso entendimento sobre a biodiversidade em florestas tropicais modificadas pelo homem ainda é incipiente, por isso, vale ressaltar que, por mais que tenha sido observada uma alta diversidade em áreas relativamente pequenas, ainda há risco de extinções locais de espécies pela perda de habitat e fragmentação. A peculiaridade das Reservas Legais unidas proporcionou a singularidade da região, e se forem feitas ações que incentivem essa prática e que possam, ainda, formar fragmentos maiores, estes poderão servir como corredores e trampolins ecológicos para as espécies, facilitando a permeabilidade e a movimentação dos indivíduos e proporcionando um maior fluxo gênico, juntamente com um aumento na variabilidade das espécies, não apenas dos escarabeíneos, mas de todos os organismos (LINHARES, 2003).

\section{Agradecimentos}

Aos proprietários que permitiram as coletas nas suas propriedades. Ao professor Dr. José Salatiel Rodrigues Pires pela ajuda na confecção dos mapas e ao Dr. Pedro Giovâni da Silva pela confirmação da identificação das espécies. MIMH agradece ao CNPq por concessão de Bolsa Produtividade em Pesquisa (Processo 309030/2013-7).

\section{Referências}

ALMEIDA, S. S. P.; LOUZADA, J. N. C. Estrutura da comunidade de Scarabaeinae (Scarabaeidae: Coleoptera) em fitofisionomias do Cerrado e sua importância para a conservação. Neotropical Entomology, Londrina, v. 38, n. 1, p. 32-43, 2009.

AUDINO, L. D.; SILVA, P. G. da; NOGUEIRA, J. M.; MORAES, L. P. de; VAZ-DE-MELLO, F. Z. Scarabaeinae (Coleoptera, Scarabaeidae) de um bosque de eucalipto introduzido em uma região originalmente campestre. Iheringia, Série Zoologia, Porto Alegre, v. 101, n. 1-2, p. 121-126, 2011.

BACK, A. J. Caracterização climática. In: MILIOLI, G.; SANTOS, R.; ZANETTE, V. C. (Ed.). Mineração de carvão, meio ambiente e desenvolvimento sustentável no sul de Santa Catarina: uma abordagem interdisciplinar. 1 ed. Curitiba: Juruá, 2009. p. 17-33.

BARLOW, J.; GARDNER, T. A.; ARAÚJO, I. S.; ÁVILAPIRES, T. C.; BONALDO, A. B.; COSTA, J. E.; ESPOSITO,
M. C.; FERREIRA, L. V.; HAWES, J.; HERNÁNDEZ, M. I. M.; HOOGMOED, M. S.; LEITE, R. N.; LO-MAN-HUNG, N. F.; MALCOLM, J. R.; MARTINS, M. B.; MESTRE, L. A. M.; MIRANDA-SANTOS, R.; NUNES-GUTJAHR, W. L.; OVERAL, A. L.; PARRY, L.; PETERS, S. L.; RIBEIRO JR., M. A.; DA SILVA, M. N. F.; DA SILVA MOTTA, C.; PERES, C. A. Quantifying the biodiversity value of tropical primary, secondary, and plantation forests. Proceedings of National Academy of Sciences of the United States of America, Washington, v. 104, n. 47, p. $18555-$ 18560, 2007.

BATILANI-FILHO, M. Funções ecossistêmicas realizadas por besouros Scarabaeinae na decomposição da matéria orgânica: aspectos quantitativos em áreas de Mata Atlântica. 2015. $91 \mathrm{f}$. Dissertação (Mestrado em Ecologia) - Universidade Federal de Santa Catarina, Florianópolis. 2015.

BETT, J. Z.; FARIAS, P. M.; SILVA, P. G. da; HERNÁNDEZ, M. I. M. Dung beetle communities in coal mining areas in the process of recovery. Biotemas, Florianópolis, v. 27, n. 3, p. 197-200, 2014. BRAGA, R. F.; KORAZAKI, V.; ANDRESEN, E.; LOUZADA, J. Dung beetle community and functions along a habitat-disturbance gradient in the Amazon: a rapid assessment of ecological functions associated to biodiversity. Plos One, San Francisco, v. 8, n. 2, 2013. e57786. doi:10.1371/journal.pone.0057786.

BRASIL. Lei no 12.651, de 25 de maio de 2012. Dispõe sobre a proteção da vegetação nativa. Brasília: Diário Oficial da República Federativa do Brasil, 2012. Disponível em: $<$ http://www.planalto. gov.br/ccivil_03/_ato2011-2014/2012/lei/L12651compilado.htm>. Acesso em: 4 mar. 2015

BROWN, K. S. JR. Diversity, disturbance, and sustainable use of Neotropical forests: insects as indicators for conservation monitoring. Journal of Insect Conservation, Headington, v. 1, p. 25-42, 1997.

CAMPOS, R. C.; HERNÁNDEZ, M. I. M. Dung beetle assemblages (Coleoptera, Scarabaeinae) in Atlantic forest fragments in southern Brazil. Revista Brasileira de Entomologia, Curitiba, v. 57, n. 1, p. 47-54, 2013.

CAMPOS, R. C.; HERNÁNDEZ, M. I. M. Changes in the dynamics of functional groups in communities of dung beetles in Atlantic forest fragments adjacent to transgenic maize crops. Ecological Indicators, Amsterdam, v. 49, p. 216-227, 2014.

CLARKE, K. R.; GORLEY, R. N. Primer V. $6 \beta$ R3. Plymouth: Plymouth Marine Laboratory, 2006.

COLWELL, R. K. EstimateS: statistical estimation of species richness and shared species from samples v. 9.1.0. 2013. Disponível em: <purl.oclc.org/estimates>. Acesso em: 31 out. 2014.

DAVIS, A. J.; HOLLOWAY, J. D.; HUIJBREGTS, H.; KRIKKEN, J.; KIRK-SPRIGGS, A. H.; SUTTON, S. L. Dung beetles as indicators of change in the forests of northern Borneo. Journal of Applied Ecology, London, v. 38, p. 593-616, 2001.

DE MARCO, Jr. P.; COELHO, F. M. Services performed by the ecosystem: forest remnants influence agricultural cultures' pollination and production. Biodiversity and Conservation, Berlin, v. 13, p. 1245-1255, 2004.

DUFRÊNE, M.; LEGENDRE, P. Species assemblages and indicator species: the need for flexible asymmetrical approach. Ecological Monographs, Washington, v. 67, n. 3, p. 345-366, 1997.

FARIAS, P. M. de. Diversidade de Scarabaeinae (Coleoptera: Scarabaeidae) em agroecossistemas: funções ecossistêmicas 
e contribuição na ciclagem de nutrientes. 2016. 183 f. Tese (Doutorado em Ecologia) - Universidade Federal de Santa Catarina, Florianópolis. 2016.

FILGUEIRAS, B. K. C.; TABARELLI, M.; LEAL, I. R.; VAZDE-MELLO, F. Z.; IANNUZZI, L. Dung beetle persistence in human-modified landscapes: combining indicator species with anthropogenic land use and fragmentation-related effects. Ecological Indicators, Amsterdam, v. 55, p. 65-73, 2015.

FORMAN, R. T. T.; GODRON, M. Landscape ecology. New York: Wiley \& Sons Ed., 1986. 640 p.

GARDNER, T. A.; BARLOW, J.; ARAUJO, I. S.; PIRES, T. C. A.; BONALDO, A. B.; COSTA, J. E.; ESPOSITO, M. C.; FERREIRA, L. V.; HAWES, J.; HERNÁNDEZ, M. I. M.; HOOGMOED, M. S.; LEITE, R. N.; LO-MAN-HUNG, N. F.; MALCOLM, J. R.; MARTINS, M. B.; MESTRE, L. A. M.; SANTOS, R. M.; OVERAL, W. L.; PARRY, L.; PETERS, S. L.; RIBEIRO-JUNIOR, M. A.; SILVA, M. N. F.; MOTTA, C. S.; PERES, C. A. The costeffectiveness of biodiversity surveys in tropical forests. Ecology Letters, Hoboken, v. 11, p. 139-150, 2008a.

GARDNER, T. A.; HERNÁNDEZ, M. I. M.; BARLOW, J.; PERES, C. A. Understanding the biodiversity consequences of habitat change: the value of secondary and plantation forests for Neotropical dung beetles. Journal of Applied Ecology, London, v. 45 , p. $883-893,2008$ b.

HALFFTER, G.; ARELLANO, L. Response of dung beetle diversity to human-induced changes in a tropical landscape. Biotropica, Hoboken, v. 34, n. 1, p. 144-154, 2002.

HALFFTER, G.; EDMONDS, W. D. The nesting behavior of dung beetles (Scarabaeinae): an ecological and evolutive approach. Ciudad de México: Man and the Biosphere Program UNESCO, 1982. 177 p.

HALFFTER, G.; FAVILA, M. E. The Scarabaeinae (Insecta: Coleoptera) an animal group for analysing, inventorying and monitoring biodiversity in tropical rain forest and modified landscapes. Biology International, Orsay, v. 27, p. 15-21, 1993.

HALFFTER, G.; FAVILA, M. E.; HALFFTER, V. A comparative study of the structure of the scarab guild in Mexican tropical rain forest and derived ecosystems. Folia Entomologica Mexicana, Texcoco, v. 84, p. 131-156, 1992.

HALFFTER, G.; MATTHEWS, E. G. The natural history of dung beetles of the subfamily Scarabaeinae (Coleoptera, Scarabaeidae). Folia Entomologica Mexicana, Texcoco, v. 12, p. 1-312, 1966.

HANSKI, I.; CAMBEFORT, Y. Dung beetle ecology. Princeton: Princeton University Press, 1991. 482 p.

HERNÁNDEZ, M. I. M.; BARRETO, P. S. C. S.; COSTA, V. H.; CREÃO-DUARTE, A. J.; FAVILA, M. E. Response of a dung beetle assemblage along a reforestation gradient in Restinga forest. Journal of Insect Conservation, Berlin, DOI 10.1007/s10841014-9645-5, 2014.

HERNÁNDEZ, M. I. M.; VAZ-DE-MELLO, F. Z. Seasonal and spatial variation of coprophagous Scarabaeidae s. str. (Coleoptera) species richness in areas of Atlantic Forest of the state of São Paulo, Brazil. Revista Brasileira de Entomologia, Curitiba, v. 53, n. 4, p. 498-505, 2009.

IBGE - INSTITUTO BRASILEIRO DE GEOGRAFIA E ESTATÍSTICA. Cidades: Içara. 2014. Disponível em: <http:// www.cidades.ibge.gov.br/painel/painel.php?lang $=\& \operatorname{codmun}=420$ 700\&search $=\mid$ infogr\%E1ficos:-dados-gerais-do-munic\%EDpio>. Acesso em: 20 nov. 2014.

KLEIN, B. C. Effects of forest fragmentation on dung and carrion beetle communities in Central Amazonia. Ecology, Washington, v. 70, n. 6, p. 1715-1725, 1989.

KORASAKI, V.; BRAGA, R. F.; ZANETTI, R.; MOREIRA, F. M. S.; VAZ-DE-MELLO, F. V.; LOUZADA, J. Conservation value of alternative land-use systems for dung beetles in Amazon: valuing traditional farming practices. Biodiversity and Conservation, Madrid, v. 22, p. 1485-1499, 2013.

LINHARES, C. A. As unidades de conservação são adequadas à preservação das espécies animais? In: SIMPÓSIO BRASILEIRO DE SENSORIAMENTO REMOTO, 5, 2003, São José dos Campos. Anais... São José dos Campos: SBSR, 2003. p. 1339-1346.

LOBO, J. M.; MARTIN-PIERA, F.; VEIGA, C. M. Las trampas pitfall con sebo, sus posibilidades en el estudio de las comunidades coprófagas de Scarabaeoidea (Col.). I. Características determinantes de su capacidad de captura. Revue d'Écologie et de Biologie du Sol, Paris, v. 25, p. 77-100, 1988.

McCUNE, B.; MEFFORD, M. J. PC-ORD multivariate analysis of ecological data v. $5.10 \mathrm{MjM}$ Software, Oregon: Gleneden Beach, 2006.

MEDRI, I. M.; LOPES, J. Scarabaeidae (Coleoptera) do Parque Estadual Mata dos Godoy e de área de pastagem, no norte do Paraná, Brasil. Revista Brasileira de Zoologia, Curitiba, v. 18, n. 1, p. 135-141, 2001.

MENDEZ, H. A. G. Influência de corredor de vegetação na riqueza e abundância de Scarabaeinae (Insecta: Coleoptera) e de parasitóides (Insecta: Hymenoptera) em um agrossistema de cafeeiro. 2007. 49 f. Dissertação (Mestrado em Entomologia Agrícola) - Universidade de Lavras, Lavras. 2007.

METZGER, J. P. O que é ecologia de paisagens? Biota Neotropica, Campinas, v. 1, p. 1-9, 2001.

METZGER, J. P. O Código Florestal tem base científica? Natureza \& Conservação, São José dos Pinhais, v. 8, n. 1, p. 92-99, 2010.

METZGER, J. P.; FONSECA, M. A. da; OLIVEIRA FILHO, F. J. B. de; MARTENSEN, A. C. O uso de modelos em ecologia de paisagens. Megadiversidade, Belo Horizonte, v. 3, n. 1, p. 64-73, 2007.

NICHOLS, E.; SPECTOR, S.; LOUZADA, J.; LARSEN, T.; AMEZQUITA, S.; FAVILA, M. E. Ecological functions and ecosystem services provided by Scarabaeinae dung beetles. Biological Conservation, Boston, v. 145, p. 1461-1474, 2008.

RESENDE, N. F. Mudanças do uso da terra no Cerrado sobre comunidade de besouros escarabeíneos (Coleoptera: Scarabaeidae: Scarabaeinae). Revista Brasileira de Gestão e Engenharia, São Gotardo, v. 5, p. 87-102, 2012.

RIBEIRO, M. C.; METZGER, J. P.; MARTENSEN, A. C.; PONZONI, F. J.; HIROTA, M. M. The Brazilian Atlantic Forest: how much is left, and how is the remaining forest distributed? Implications for conservation. Biological Conservation, Boston, v. 142, p. 1141-1153, 2009.

RICKLEFS, R. E. A economia da natureza. Rio de Janeiro: Guanabara Koogan, 2011. 546 p.

RIEFF, G. G.; MACHADO, R. G.; STROSCHEIN, M. R. D.; DE SÁ, E. L. S. Diversidade de famílias de ácaros e colêmbolos 
edáficos em cultivo de eucalipto e áreas nativas. Revista Brasileira de Agrociência, Pelotas, v. 1, n. 1-4, p. 57-61, 2010.

SALA, O. E.; CHAPIN III, S. F.; ARMESTO, J. J.; BERLOW, E.; BLOOMFIELD, J.; DIRZO, R.; HUBER-SANWALD, E.; HUENNEKE, L. F.; JACKSON, R. B.; KINZING, A.; LEEMANS, R.; LODGE, D. M., MOONEY, H. A.; OESTERHELD, M.; POFF, L. R. N.; SYKES, M. T.; WALKER, B. H.; WALKER, M.; WALL, D. H. Global biodiversity scenarios for the year 2100. Science, Washington, v. 287, p. 1770-1774, 2000.

SCHIFFLER, G.; VAZ-DE-MELLO, F. Z.; AZEVEDO, C. O. Scarabaeidae s.str. (Coleoptera) do Delta do Rio Doce e Vale do Suruaca no município de Linhares, estado do Espírito Santo, Brasil. Revista Brasileira de Zoociências, Juiz de Fora, v. 5, n. 2, p. $205-$ 211, 2003.

SILVA, P. G. da; HERNÁNDEZ, M. I. M. Spatial patterns of movement of dung beetle species in a tropical forest suggest a new trap spacing for dung beetle biodiversity studies. Plos One, San Francisco, v. 10, n. 5, p. 1-18, 2015.

SILVA, P. G. da; VAZ-DE-MELLO, F. Z.; DI MARE, R. A. Diversity and seasonality of Scarabaeinae (Coleoptera: Scarabaeidae) in forest fragments in Santa Maria, Rio Grande do Sul, Brazil. Anais da Academia Brasileira de Ciências, Rio de Janeiro, v. 85, n. 2, p. 679-697, 2013.

SILVA, R. J.; COLLETTI, F.; COSTA, D. A.; VAZ-DE-MELLO, F. Z. Rola-bostas Coleoptera: Scarabaeidae: Scarabaeinae) de florestas e pastagens no sudoeste da Amazônia brasileira: levantamento de espécies e guildas alimentares. Acta Amazonica, Manaus, v. 44, n. 3, p. 345-352, 2014.
SIMÕES, T. R. O. Diversidade de besouros indicadores em fragmentos florestais nativos e exóticos na região de Anitápolis - Santa Catarina, Brasil. 2013. 63 f. Monografia (Trabalho de Conclusão de Curso em Ciências Biológicas) - Universidade Federal Santa Catarina, Florianópolis. 2013.

SIZENANDO, J. W. Mineração e espacialidade socioambiental: exploração carbonífera e ocupação territorial em Criciúma, Santa Catarina. 2011. 73 f. Dissertação (Mestrado em Ciências Ambientais) - Universidade do Extremo Sul Catarinense, Criciúma. 2011.

SLADE, E. M.; MANN, D. J.; VILLANUEVA, J. F.; LEWIS, O. T. Experimental evidence for the effects of dung beetle functional group richness and composition on ecosystem function in a tropical forest. Journal of Animal Ecology, London, v. 76, p. 1094-104, 2007.

SOULÉ, M. E. Conservation: tactics for a constant crisis. Science, Washington, v. 5021, p. 744-750, 1991.

TABARELLI, M.; AGUIAR, A. V.; RIBEIRO, M. C.; METZGER, J. P.; PERES, C. A. Prospects for biodiversity conservation in the Atlantic Forest: lessons from aging human-modified landscapes. Biological Conservation, Boston, v. 143, p. 2328-2340, 2010.

THOMAZINI, M. J.; THOMAZINI, A. P. B. W. A fragmentação florestal e a diversidade de insetos nas Florestas Tropicais Úmidas. Rio Branco: EMBRAPA, Acre, (Documentos 57), 2000. $21 \mathrm{p}$. 